\title{
Anterior esthetic fixed appliance in pediatric patient: case report
}

Authors

\section{Alsafran Faisal A, Albraithen, Abdulrahman A, Bin Talib Bader S}

\section{Introduction}

ECC, when associated with the bottle habit, has been characterized as first affecting the primary maxillary anterior teeth, followed by involvement of the primary molars. The extent of decay is almost always more severe in the maxillary incisors, and, frequently, by the time the child is brought to the dentist, much of the anterior clinical crowns are decayed or lost.

When extraction of primary incisors is necessary, many parents will seek an esthetic solution to replace the lost teeth. Parental desire is one of the main decisive factors for treating these types of clinical situations.

\section{Case Report}

4 years and 6 months girl came to pediatric dentistry screening clinic at Imam Abdulrahman Alfaisal hospital, Dammam, Saudi Arabia with a chief complaint as stated by the mother "I want to restore my daughter's carious teeth". Mother mentioned that dental caries started when the girl was 2 years old and this is the first dental visit for her. Regarding oral hygiene, mother mentioned that the girl does not brush her teeth regularly and usually without parental supervision, consumed a lot of sugary snacks daily, a history of bedtime bottle habit.
Upon intra-oral examination, patient has poor oral hygiene with multiple carious teeth. The upper incisors was extensively decayed and indicated for extraction.

Oral rehabilitation under general anesthesia was the option of treatment chosen due to extensive operative procedures, young age of the patient with uncooperative behavior in the screening appointment.

Initial treatment plan discussed with mother, and she asked for replacement of the upper incisors after extractions. The final treatment plan postponed until radiographs taken in the OR prior to oral rehabilitation.

In the operation day, after the patient anesthetized with general anesthesia, full mouth radiographs and photographs taken and final treatment plan decided.

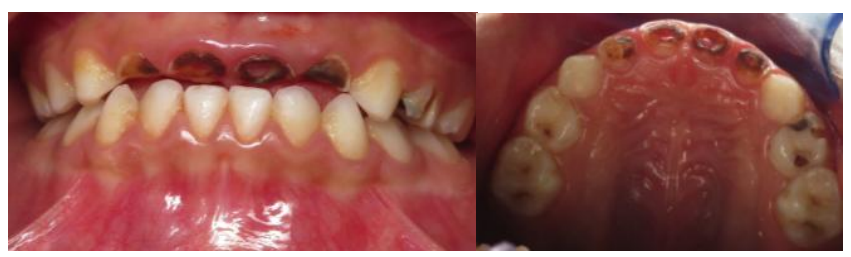

Pre-operative photographs 
Oral prophylaxis and rubber dam isolation applied, the restorative work of molars and canines including SSCs, amalgam and composite restorations completed.

Then, the upper four incisors extracted. Band selected for \#55, 65 and upper and lower alginate impression taken, bite registered. Fluoride gel applied.

Ten days post operatively; the patient came for postoperative follow-up, the restorations, crowns, soft tissue and oral hygiene evaluated, oral hygiene instructions given and less cariogenic diet recommended.

Four weeks post operatively, the anterior esthetic fixed appliance cemented with glass ionomer cement, and recall appointment after two months given.

Three months post operatively; the patient came for recall visit. Oral exam revealed that the appliance is stable and the patient and the mother is satisfying from the outcome result. Fluoride gel applied and recall appointment after three months given.

Six months post operatively; the patient came for recall visit. Oral exam revealed that the appliance is stable and the patient and the mother is satisfying from the outcome result. Oral hygiene is good with no caries. Fluoride gel applied, radiographs and photographs taken and recall appointment after three months given.

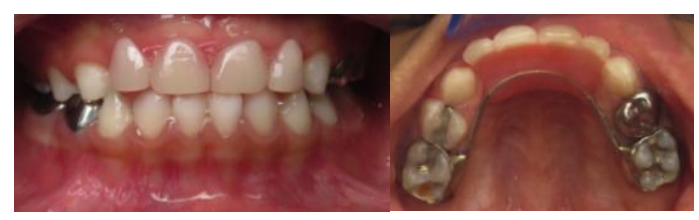

6 months recall photographs

\section{Discussion}

Premature loss of maxillary primary anterior teeth may lead to psychological disturbances and affect the child's self-esteem and socialization during the important phase of development. One of the most important and valid reasons for replacing missing incisors is to restore a natural and pleasing appearance and thus provide an opportunity for normal psychological development.

While space maintenance in the posterior region is an important consideration when there is early loss of primary molars, the anterior segment, from canine to canine, appears to be stable, even with the early loss of several incisors. Parents may express concern about their child's ability to eat without four incisors. They need to be reassured that feeding is generally not a problem. Children who have had all four maxillary incisors extracted due to EEC seem to function well without them. Yet another consideration is the child's speech development following extraction of all four incisors. Children over 4 years will usually compensate for the tooth loss and not exhibit any long-term speech disorders.

Plaque and food debris accumulation is increased with the fixed anterior appliance. Many children who had all incisors extracted are highly susceptible to caries risk due to EEC. It is imperative that the parents understand the risks involved in treating their child. A comprehensive caries prevention program must be initiated with a frequent recall schedule. Adequate measures of oral hygiene and a modified less-cariogenic diet should be achieved before placing any appliance in the mouth. Limitations like long-term followup, improper oral hygiene maintenance, and frequent breakage can be decreased by proper education and motivation of the child and the parents.

After all, If parents do not indicate a desire to replace missing anterior teeth, no treatment is usually required. But, if the parents do wish to replace missing teeth, they should not be discouraged from their decision.

\section{Conclusion}

The placement of an anterior primary fixed appliance is an elective procedure and is based strongly on parental desires. Parents must be able to make an informed decision and the pediatric dentist should provide them with accurate information facilitating such a decision. 


\section{References}

1. William F. Waggoner DDS, MS Ari Kupietzky DMD, MSc. Anterior esthetic fixed appliances for the preschooler: considerations and a technique for placement. Pediatric Dentistry - 23:2, 2001

2. Diptangshu Garai, Chiranjit Ghosh, Pradip Kumar Mandal, SudiptaKar. Esthetic Anterior Fixed Functional Space Maintainer. International Journal of Pedodontic Rehabilitation | Volume 2 ! Issue 2 ! July-December 2017

3. K. P. Aswanth, Sharath Asokan, Baby John J. Fixed functional space maintainer: A weight gainer: A case report. Journal of Indian Academy of Dental Specialist Researchers | Vol. 1 | Issue 1 | Jan-Jun 2014

4. Waggoner WF, Kupietzky A. Anterior esthetic fixed appliances for the preschooler: Considerations and a technique for placement. Am AcadPediatr Dent 2001;23:2.

5. Koroluk LD, Riekman GA. Parental perceptions of the effects of maxillary incisor extractions in children with nursing caries. J Dent Child 58:233-236, 1991.

6. Aswanth KP, Asokan S, John BJ. Fixed functional space maintainer: A weight gainer: A case report. J Indian Acad Dent Spec Res 2014;1:25-7. 